\title{
Detonation Limits in Rough Walled Tubes
}

\author{
Amanda Starr ${ }^{1}$, John H.S. Lee ${ }^{1}$ and Hoi Dick $\mathrm{Ng}^{2}$ \\ ${ }^{1}$ Department of Mechanical Engineering, \\ McGill University, Montreal, Quebec, H3A 2K6, Canada \\ Fax: 514-398-7365 \\ ${ }^{2}$ Department of Mechanical and Industrial Engineering, \\ Concordia University, Montreal, Quebec, H3G 1M8, Canada \\ Email Addresses: amanda.starr@mail.mcgill.ca (Amanda Starr); \\ john.lee@mcgill.ca (John Lee); \\ hoing@encs.concordia.ca (Hoi Dick Ng)
}

Colloquium: DETONATIONS, EXPLOSIONS and SUPERSONIC COMBUSTION Total length of paper: 6114 words using Method 1 Word equivalent lengths:

Main text: 3127, Equations: 0, Nomenclature: 0, References: 630 ,Tables: 0 Figures and Captions: Total: 2357

Fig.1: 247, Fig.2: 95, Fig.3: 149, Fig.4: 139

Fig.5: 136, Fig.6: 644, Fig.7: 641, Fig.8: 153, Fig.9: 153 


\section{Abstract}

The present paper reports the results of a study of detonation limits in rough tubes. Detonation velocity is measured by photodiodes and ionization probes spaced at $10 \mathrm{~cm}$ intervals along the length of the tube. Short lengths of smoked foils inserted into the core of the rough tube is used to register the structure of the detonation wave. Pressure transducers are also used to obtain the pressure profile. The results indicate that in rough tubes, the detonation velocity is generally much lower than the corresponding values for smooth tubes. The velocity decreases slowly at first and then more rapidly as the limit is approached. The velocity variation is generally continuous and at the limits, the failure velocity is of the order of about 0.4 $V_{C J}$ for all cases. The detonation limits in rough tubes are found to be wider than for a smooth tube. This indicates that the turbulence generated by the wall roughness facilitates the propagation of the detonation and extends the limits. Smoked foil records show that in the core of the rough tube the detonation front has a cellular structure corresponding to the usual cellular structure due to instability of the detonation. Thus the intrinsic unstable cellular structure is quite robust and retains its global characteristics in spite of the large perturbations generated by the rough wall. The detonation in the core of the rough tube goes from multi-headed to single headed as the limit is approached. Past the single headed spin, the low velocity detonation has no cellular structure but consists of interacting weak transverse waves from the rough wall. The averaged pressure of the low velocity detonation front corresponds to about the constant volume explosion pressure, in accord with the velocity of the low velocity detonation.

Keywords: Detonation Limit, Turbulence. 


\section{Introduction}

Existing investigations of detonation limits have all been carried out in smooth circular tubes. The general behavior of the detonation as the limits are approached is a decrease in the velocity and an increase in the cell size. In smooth tubes, the detonation velocity seldom drops below $80 \%$ of the Chapman-Jouguet velocity $\left(V_{C J}\right)$ when failure occurs . In certain mixtures and tube diameters, the detonation also manifests longitudinal oscillations just prior to failure (i.e., large amplitude fluctuations in the propagation velocity). These are referred to as "stuttering" and "galloping" detonations. The failure mechanism at the detonation limit is not known. In the early theory of Zel'dovich [1], momentum and heat losses were considered responsible for increasing the velocity deficit that eventually led to failure. On the other hand, recent studies indicate that the suppression of the instability of the detonation front can also lead to failure [2]. When the transverse waves associated with cellular instability of the detonation front are attenuated in a porous walled tube, the detonation fails [3]. Since almost all detonations are unstable, it is reasonable to assume that cellular instability is required for the self-sustained propagation of detonation waves. Note that as the limits are approached, the cellular instability tends towards lower transverse modes in general until single headed spin is obtained prior to failure. Only in certain cases, when longitudinal unstable modes of "stuttering" and "galloping" detonations are observed past the single headed spin. The reason that instability is required for self-sustained propagation is not fully understood. It was suggested that the turbulent reaction zone of cellular detonation promotes mixing and hence facilitates ignition. Therefore,suppression of the cellular instability in essence suppresses turbulence in the reaction zone and this led to detonation failure. It should be noted that for high speed compressible supersonic flow in an obstacle filled tube, "turbulence" consists of strong pressure as well as velocity fluctuations. Interactions of shock waves with vorticity and density gradients provide dominant vorticity generation in contrast with classical shear flow turbulence. On the other hand, turbulence is generated artificially in rough walled tubes, and does not depend on instability. Indeed it is found that detonation propagation is facilitated in rough walled tubes although wall roughness results in a decrease in the detonation velocity. Hence if turbulence generated

artificially in rough walled tubes can facilitate detonation propagation, then it is reasonable to assume that the detonation limits should also be wider in rough walled tubes. The influence of wall roughness on detonation limits has not been investigated to date. It is thus of interest to determine if turbulence can extend 
the detonation limits.

The study of detonation in rough tubes was first carried out by Laffitte [4] and later Shchelkin [5] and Chapman and Wheeler [6] whose interest is to promote the deflagration-to-detonation transition (DDT). Although the understanding of DDT in rough tubes still remains one of the major challenges of detonation theory, significant insights have been gained through a number of recent studies. Notably, using highresolution numerical simulations Gamezo et al. [7-9] investigate the effect of obstacles and their different arrangement on DDT. Their numerical results reproduce the main regimes of flame propagation observed in experiment such as supersonic turbulent flames, the abrupt onset of detonation and its subsequent propagation. The review paper by Oran and Gamezo [10] also discusses the computational challenges in obtaining a quantitative prediction of DDT. Besides the strong turbulence and hydrodynamic fluctuations created by the obstacles which increase the burning rate and facilitates the flame acceleration, few analytical studies have also proposed other possible physical mechanism for DDT in rough tubes such as the concept of hydraulic resistance [11,12], the delayed burning and jet generation mechanism [13,14]. More recent experimental results on flame acceleration and DDT in tubes with repeated obstacles or multi-bend geometry have been reported [15-18] and a comprehensive review on the subject can also be found in the papers by Ciccarelli and Dorofeev [19], Dorofeev [20] and references therein.

Apart from DDT, Guénoche [21] however, carried out a systematic study and measured the steady state velocity of detonations in rough tubes. Also, of interest is the study by Manson et al. [22] where streak Schlieren photographs of detonation in rough tubes were taken illustrating the effect of the artificially induced transverse waves by the wall roughness on the intrinsic transverse instability of the cellular detonations. A number of subsequent studies also concern with the structure and propagation mechanism of quasi-detonations and fast turbulent deflagrations in rough obstacle-filled tubes, e.g. [23-29].

Preliminary study of detonation limits in rough walled tubes has been reported recently [30]. Since then, more extensive experiments with added diagnostics of smoked foils and pressure transducers are used to determine the cellular detonation structure and pressure profiles. The present paper reports these recent findings. 


\section{Experimental Details}

Detonation tubes of diameters $D=12.7 \mathrm{~mm}$ and $D=50.8 \mathrm{~mm}$ were used in the present study. Fig. 1 shows a schematic of the experimental set-up. A $1 \mathrm{~m}$ long steel driver section using a more detonable gas is used for initiating the detonation in the test section. The test section is $1.5 \mathrm{~m}$ long and the obstacle (rough) section is $1 \mathrm{~m}$, giving an initial $0.5 \mathrm{~m}$ length of a smooth section to provide a reference detonation velocity prior to its entry into the rough section. It was found that the detonation adjusts rapidly (within about two tube diameters in general) upon entering the rough section. Thus it suffices to use only a 1 $\mathrm{m}$ long test section. It is important to note that when the initial pressure is lowered past the limit in the smooth tube determined by Gao et al. [31], it is necessary to extend the rough section all the way to the driver.

A Shchelkin spiral of pitch of one tube diameter was used to generate the wall roughness. Previous investigations indicated that a pitch about one tube diameter is the most effective roughness and also it was found that the phenomenon is not too sensitive to the pitch of the spiral. The diameters of the wire of the spiral used were $\delta=1.6$ and $3.2 \mathrm{~mm}$ for the $12.7 \mathrm{~mm}$ tube and for the larger tube of $50.8 \mathrm{~mm}$, the wire diameters used were $\delta=6.4$ and $9.5 \mathrm{~mm}$. The ratio of the wire diameter to the tube diameter $\delta / D$ was used to characterize the wall roughness of the Shchelkin spiral. The spiral characteristics are shown in Fig. 2.

Pre-mixed mixtures of $\mathrm{C}_{2} \mathrm{H}_{2}+2.5 \mathrm{O}_{2}+70 \%$ Ar dilution and $\mathrm{CH}_{4}+2 \mathrm{O}_{2}$ were used. The former mixture represented a so-called "stable" mixture where the detonation has a regular cellular pattern. The methane mixture represents an "unstable" mixture with an irregular cellular pattern. Equimolar $\mathrm{C}_{2} \mathrm{H}_{2}+$ $\mathrm{O}_{2}$ (a readily detonable mixture) is filled into the driver section to promote the initiation of a detonation in the less sensitive test mixture. The mixture in the driver section is detonated by a spark from a high voltage discharge of a low inductance capacitor $(15 \mathrm{kV}$ and $0.2 \mu \mathrm{F})$.

Velocity measurement was made by regularly spaced photodiodes $(10 \mathrm{~cm}$ apart) along the length of the test section. The photodiodes were more closely spaced near the beginning of the coil as to capture the 
adjustment of the detonation upon entering the rough section. An ionization probe was also used to supplement the photodiode signals when the light from the detonation front becomes weak near the limits. A PCB pressure transducer (model 113A24) was also used to monitor the detonation pressure signal near the limit. Smoked foils were employed to record the cellular structure of the detonation near the limits. The smoke foil is located near the end of the test section. The foil captures only the detonation core since it is inserted into the inner diameter of the spiral. Although the smoked foil shields the detonation from the roughness at the wall, it is found that the detonation structure is retrained for some distance of travel after entering the smoked foil section. Thus the foil can register the detonation structure in the rough tube.

\section{Results and Discussions}

For a given mixture, tube diameter and roughness $(\delta / D)$, the detonation limits are approached by progressively lowering of the initial pressure. An overdriven detonation is produced in the driver section which decays to a self-propagating detonation in the test section. From the photodiode and ion probe signals, the detonation trajectory can be obtained. Fig. 3 shows the detonation trajectories for $\mathrm{C}_{2} \mathrm{H}_{2}+2.5 \mathrm{O}_{2}+70 \% \mathrm{Ar}$ in a $12.7 \mathrm{~mm}$ diameter tube with roughness of $\delta / D=0.13$. Far from the limit (12 $\mathrm{kPa})$, prior to entry into the rough section of the tube, the detonation velocity is about $1626 \mathrm{~m} / \mathrm{s}$ corresponding to a typical velocity deficit of about $5 \%$ for the mixture. Note all CJ calculations are computed using the NASA CEA program [32]. Subsequent to entering the rough section, the detonation velocity drops rapidly and reaches a lower steady state value. The velocity in the rough section decreases slowly with decreasing initial pressure, and more rapidly as the limits are approached. Near the limiting pressure itself, the light intensity of the detonation front is often very weak and a number of photodiode signals are missing. However an ion probe will give a signal when a combustion arrives at its location. Combining with the photodiode signals, it is possible to determine if a steady detonation is obtained or if the detonation fails . Past the limiting pressure $(1.5 \mathrm{kPa})$, we note from the long time delay registered by the ion probe signal (denoted by the closed diamond symbols) that the detonation wave fails and a deflagration with much reduced velocity is obtained.

Fig. 4 shows the variation of the detonation velocity with the ratio of the diameter " $d$ " to the cell size 
" $\lambda$ " for $\mathrm{C}_{2} \mathrm{H}_{2}+2.5 \mathrm{O}_{2}+70 \%$ Ar. The detonation cell sizes are determined using the CALTECH detonation database [33]. The diameter " $d$ " for the rough tube is given by $d=D-2 \delta$, where $\delta$ is the wire diameter of the Shchelkin spiral. Thus " $d$ " represents the central core diameter of the rough tube. Also shown for comparison in Fig.4 are the results for a smooth tube obtained by Gao et al. [31]. For the smooth tube, the results for both tube diameters of $12.7 \mathrm{~mm}$ and $50.8 \mathrm{~mm}$ lie on a single curve using the dimensionless ratio $d / \lambda$. Note that the detonation velocity decreases slowly from about $V / V_{C J} \cong 0.95$ to about $V / V_{C J} \cong 0.8$ when failure occurs in the smooth tube. The value of $d / \lambda \cong 0.2$ at the limit which closely corresponds to that of a single headed spinning detonation where $d / \lambda \cong 1 / \pi$. For the $12.7 \mathrm{~mm}$ and $50.8 \mathrm{~mm}$ diameter rough tubes, again the velocity results can be represented by a single curve. Far from the limits, the velocity $V / V_{C J} \cong 0.85$ and decreases slowly with decreasing initial pressure (or $d / \lambda$ ). It is interesting to note that there is a small abrupt decrease in the detonation velocity occurring at a value of $d / \lambda \cong 0.4$, which also corresponds to approximately the value for a single headed spinning detonation wave occurring in the core of the rough tube. Thus it appears there is a small drop in the detonation velocity when the detonation front in the core of the tube goes past the single headed spin condition. Subsequent to the small abrupt drop in the velocity, further decrease in the initial pressure (or $d / \lambda)$ indicate further decrease in the detonation velocity eventually to a value about $V / V_{C J} \cong 0.4$ when failure occurs.

For the $12.7 \mathrm{~mm}$ tube and the greatest roughness $\delta / D=0.25$, no abrupt decrease in detonation velocity is observed and the detonation velocity varies from $V / V_{C J} \cong 0.7$ to about $V / V_{C J} \cong 0.35$ when failure occurs. Thus we observe a low velocity detonation regime throughout for this case.

For unstable $\mathrm{CH}_{4}+2 \mathrm{O}_{2}$ mixtures, the velocity in both the $12.7 \mathrm{~mm}$ and $50.8 \mathrm{~mm}$ diameter tubes can also be represented by a single curve, as shown in Fig.5. The velocity decreases slowly with decreasing pressure until failure occurs abruptly at a value of about $d / \lambda \cong 0.35$. This corresponds closely to the estimated value of $d / \lambda \cong 0.314$ for a single headed spin (note again that $d=D$ for a smooth tube). For the $50.8 \mathrm{~mm}$ diameter tubes with $\delta / D=0.13$ and 0.19 , the velocity decreases very slowly until near the limit when it decreases more rapidly from a value of $V / V_{C J} \cong 0.8$ to $V / V_{C J} \cong 0.4$ when the detonation fails. The velocity curve in the $12.7 \mathrm{~mm}$ diameter tube with a $\delta / D=0.13$ coincides with that of the $50.8 \mathrm{~mm}$ tube. However for the $12.7 \mathrm{~mm}$ tube with a larger $\delta / D=0.25$, the velocity is much lower, dropping from 
a value of $V / V_{C J} \cong 0.65$ to about $V / V_{C J} \cong 0.35$ when failure occurs. Note that for the unstable $\mathrm{CH}_{4}+$ $2 \mathrm{O}_{2}$ mixture, there is no small step decrease in the velocity at the onset of single headed spin. The velocity varies continuously until failure occurs. This could be due to the fact that for an unstable mixture, the effect of the perturbations generated by the wall roughness have a more profound influence on the cellular structure of the front in the core of the tube.

From the results shown in Fig.4 and Fig.5 it is clear that wall roughness extends the limit much beyond that of the smooth tubes. For the unstable $\mathrm{CH}_{4}+2 \mathrm{O}_{2}$ mixture, it is observed that the wall roughness is much more effective in prolonging the self-sustained propagation of the detonation wave than for the stable $\mathrm{C}_{2} \mathrm{H}_{2}+2.5 \mathrm{O}_{2}+70 \%$ Ar mixture.

Note that the detonation velocity depends on the energetics of the mixture and hence does not provide information regarding the structure of the detonation front. Thus smoked foils are used to record the detonation structure in the rough tube as the limits are approached. Fig.6 shows the smoked foil record at various values of initial pressure $\left(P_{o}\right)$ for the "stable" $\mathrm{C}_{2} \mathrm{H}_{2}+2.5 \mathrm{O}_{2}+70 \%$ Ar mixture. Note that the detonation propagates from right to left. At $P_{o}=10 \mathrm{kPa}(d / \lambda \cong 2.4)$, far from the limit, we see the transformation from double to single headed spin. Closer to the limit at $P_{o}=6 \mathrm{kPa}(d / \lambda \cong 1.2)$, the single headed spin is failing and at $P_{o}=2 \mathrm{kPa}(d / \lambda \cong 0.3)$ we only observe transverse waves generated by the roughness of the tube superimposed on the faint decaying single headed spin. Further decrease in the initial pressure to the limit shows just faint random wave pattern of pressure waves due to the spiral.

Fig.7 shows a similar smoked foil record for the "unstable" $\mathrm{CH}_{4}+2 \mathrm{O}_{2}$ mixture. Away from the limit, at $P_{o}=10 \mathrm{kPa}(d / \lambda \cong 0.5)$, we see a multi-headed structure but there is an indication of the onset of single headed spin. At $P_{o}=6 \mathrm{kPa}(d / \lambda \cong 0.3)$, the single headed spin structure becomes more apparent. At $P_{o}$ $=2 \mathrm{kPa}(d / \lambda \cong 0.08)$, again the single headed spin decayed and only random waves from the spiral are recorded.

A pressure transducer is also mounted in the tube to record the pressure profile as the detonation approaches the limits in the rough tube. Fig. 8 shows the typical single headed spin pressure profile in $\mathrm{CH}_{4}$ 
$+2 \mathrm{O}_{2}$ at $P_{o}=6 \mathrm{kPa}(d / \lambda \cong 0.4)$ in the $50.8 \mathrm{~mm}$ diameter tube with $\delta / D=0.13$. This is in accordance to the smoked foil, where a single headed spin wave is obtained in the core of the rough tube. The intrinsic cellular instability of the detonation front appears sufficiently robust and is not significantly affected even with large perturbations generated by the Shchelkin spiral. From the smoked foil records we note that the path to failure is still from multi-headed to single headed spin structure then to low velocity detonation in the core of the rough tube. The "low velocity" detonation structure consists of just random transverse waves representing the pressure fluctuations generated by the wall roughness. The "low velocity" detonation prior to failure in a rough tube is very similar to a highly turbulent wave where rapid mixing due to pressure and velocity fluctuations maintain ignition and rapid combustion to permit self-sustained propagation of the combustion wave. The pressure signature of a "low velocity" detonation is shown in Fig.9 at $P_{o}=4 \mathrm{kPa}(d / \lambda \cong 0.25)$ where we can see an extended reaction zone with pressure fluctuations, but an averaged pressure that has a value slightly lower than the constant volume explosion pressure $P_{C V}$. This is in accord with velocity of about $0.5 V_{C J}$ for these low velocity detonations.

\section{Conclusions}

The present results indicate that in rough tubes, detonation velocity can vary continuously from close to the theoretical Chapman-Jouguet value (as in smooth tubes) far from the limits to about $0.4 V_{C J}$ where the detonation fails. Contrast to the detonations in smooth tubes where the detonation velocity seldom decreases to less than $0.8 V_{C J}$ at the limits. There is strong evidence that wall roughness tends to facilitate the self-sustained propagation of detonation waves. It has been already established that wall roughness facilitates the transition from deflagration to detonation [34]. The mechanism in promoting detonation propagation is due to the turbulence generated by the rough wall. The detonation structure in a rough tube is not unlike the reaction zone of a cellular detonation where pressure waves and velocity fluctuations arise from the intrinsic instability of the detonation front. Thus wall roughness promotes the generation of pressure and vorticity fluctuations and hence extends the detonation limits of smooth tubes where turbulence can only arise from the instability of the detonation front. The smoked foil records indicate that in rough tubes, the cellular instability of the detonation still occurs in the core away from the roughness near the wall. However, when conditions are such that when the natural instability cannot be sustained, turbulence 
generated by the walls takes over and maintains self-propagation of the detonation. With turbulent mixing between the reaction gases and the unburned mixture, ignition does not have to depend on adiabatic shock heating. Thus the detonation velocity can decrease to values as low as $0.4 V_{C J}$ and still the detonation continues to propagate. With turbulence generated by wall roughness, a high detonation velocity is thus not a prime requirement for ignition. For rough tubes, the determination of the detonation velocity now requires the description of the turbulent structure of the wave.

\section{Acknowledgments}

This work is supported by the Natural Sciences and Engineering Research Council of Canada (NSERC).

\section{References}

[1] Y.B. Zel'dovich, J. Exp. Theor. Phys. 10 (1940) 542-568.

[2] G. Dupré, R. Knystautas, J.H. Lee, Prog. Astronaut. Aeronaut.106 (1986) 244-259.

[3] J.H. Lee, Dynamic structure of gaseous detonation. in: A.A. Borissov (Ed.), Dynamic Structure of Gaseous Detonation in Dispersed Media, Kluwer Academic Publishers, 1991, p.1-25.

[4] P. Lafitte, C.R. Acad. Sci. 176 (1923) 1392-1395.

[5] K. Shchelkin, J. Exp. Theor. Phys. 10 (1940) 823-827.

[6] W. R. Chapman, R. V. Wheeler, J. Chem. Soc. 129 (1926) 2139-2147.

[7] V.N. Gamezo, T. Ogawa, E.S. Oran, Proc. Combust. Inst. 31 (2007) 2463-2471.

[8] V.N. Gamezo, T. Ogawa, E.S. Oran, Combust. Flame 155 (2008) 302-315.

[9] T. Ogawa, V.N. Gamezo, E.S. Oran, J. Loss Prev. Proc. Indust. 26(2013) 295-301.

[10] E.S. Oran, V.N. Gamezo, Combust. Flame 148 (2007) 4-47.

[11] I. Brailovsky, G.I. Sivashinsky, Combust. Flame 122 (2000) 492-499.

[12] G.I. Sivashinsky, Proc. Combust. Inst. 29 (2002) 1737-1761.

[13] V. Bychkov, D. Valiev, L.E. Eriksson, Phys. Rev. Lett. 101 (2008) 164501.

[14] D. Valiev, V. Bychkov, V.y. Akkerman, C.K. Law, L.E. Eriksson, Combust. Flame 157 (2010) 10121021. 
[15] C.T. Johansen, G. Ciccarelli, Combust. Flame 156 (2009) 405-416.

[16] G. Ciccarelli, C.T. Johansen, M .Parravani, Combust. Flame 157 (2010) 2125-2136.

[17] T. Obara, T. Kobayashi, S. Ohyagi, Shock Waves 22 (2012) 627-639.

[18] M.C. Gwak, J.J. Yoh, Int. J. Hydrogen Energy 38 (2013) 11446-11457.

[19] G. Ciccarelli, S.B. Dorofeev, Prog. Energy Combust. Sci. 34 (2008) 499-550.

[20] S.B. Dorofeev, Proc. Combust. Inst. 33 (2011) 2161-2175.

[21] H. Guénoche, N. Manson, Rev. de IInst. Francais de Petrole 2 (1949) 53-69.

[22] N. Manson, C. Brochet, J. Brossard, Y. Pujol, Proc. Combust. Inst. 9 (1963) 461-469.

[23] J.H. Lee, R. Knystautas, C.K. Chan, Proc. Combust. Inst. 20 (1984) 1663-1672.

[24] O. Peraldi, R. Kystautas, J.H.S. Lee, Proc. Combust. Inst. 21 (1986) 1629-1637.

[25] A. Teodorczyk, J.H.S. Lee, R. Knystautas, Proc. Combust. Inst. 22 (1988) 17231731.

[26] C.K. Chan, D.R. Greig, Proc. Combust. Inst. 22 (1988) 1733-1739.

[27] A. Teodorczyk, J.H.S. Lee, R. Knystautas. Prog. Astro. Aero. 138 (1990) 223-240.

[28] M.S. Kuznetsov, V.I. Alekseev, A.V. Bezmelnitsyn, W. Breitung, S.B. Dorofeev, I.D. Matsukov, A. Veser, Yu. G. Yankin, Effect of obstacle geometry on behavior of turbulent flames. Report FZKA-6328 (1999) Karlsruhe: Forschungszentrum Karlsruhe GmBH, Karlsruhe.

[29] J.Chao, J.H.S. Lee, Shock Waves 2002, 12(4)277-289.

[30] A. Starr, J.H.S Lee, Detonation limits in rough walled tubes. in: Proc. 24th Int. Colloquium on the Dynamics of Explosions and Reactive Systems, Taipei, Taiwan, July 28-Aug. 2, 2013.

[31] Y. Gao, J.H.S Lee, H.D. Ng, Velocity fluctuations near the detonation limits. Combust. Flame (2014), Accepted.

[32] B.J. McBride and S. Gordon, Computer program for calculation of complex chemical equilibrium compositions and applications II, Users manual and program description. NASA Rep.RP-1311-P2, 1996. [33] M. Kaneshige, J.E. Shepherd, Detonation Database, GALCIT Technical Report FM97, available at http://www.galcit.caltech.edu/detn_db/html/db.html

[34] J.E. Shepherd, J.H.S. Lee, In: M.Y. Hussaini, A. Kumar, R.H. Voigt, (eds), Major Research Topics in Combustion, Springer-Verlag, New York, 1992, p.439. 


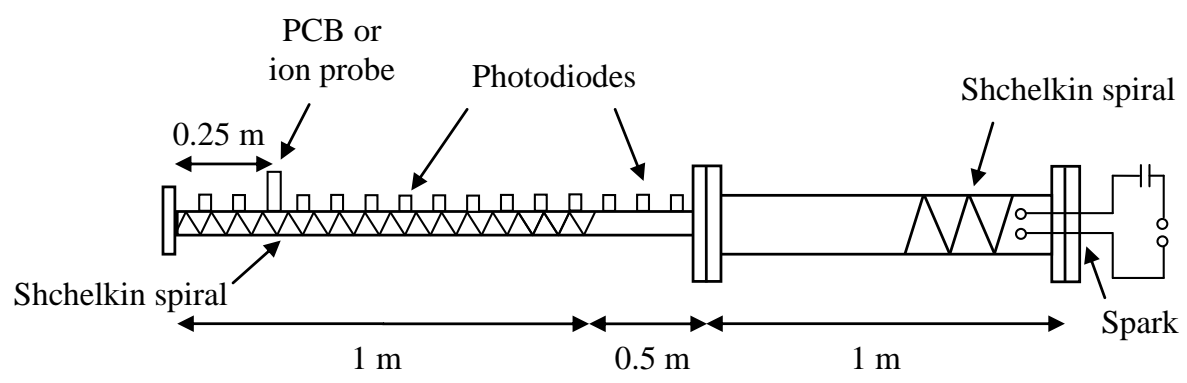

Figure 1: Schematic of the experimental setup 


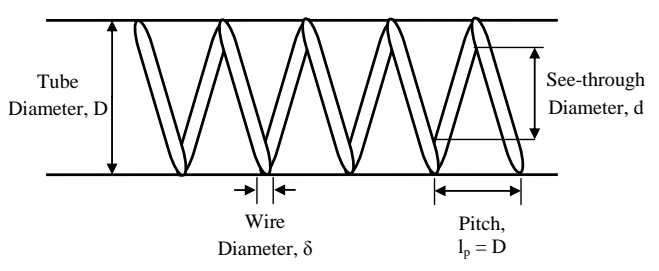

Figure 2: Wall roughness 


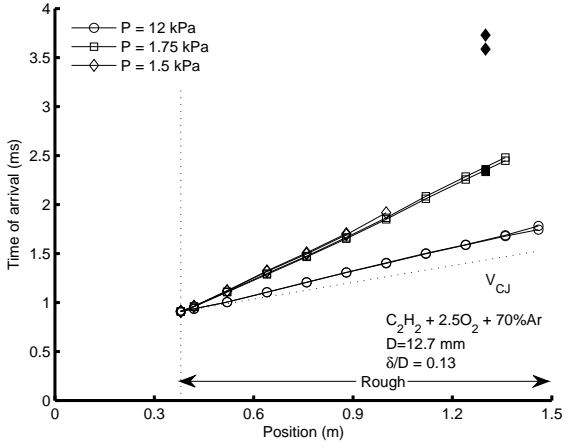

Figure 3: Detonation trajectories for $\mathrm{C}_{2} \mathrm{H}_{2}+2.5 \mathrm{O}_{2}+70 \% \mathrm{Ar}$ in the 12.7 mm diameter tube with $\delta / D=0.13$ 


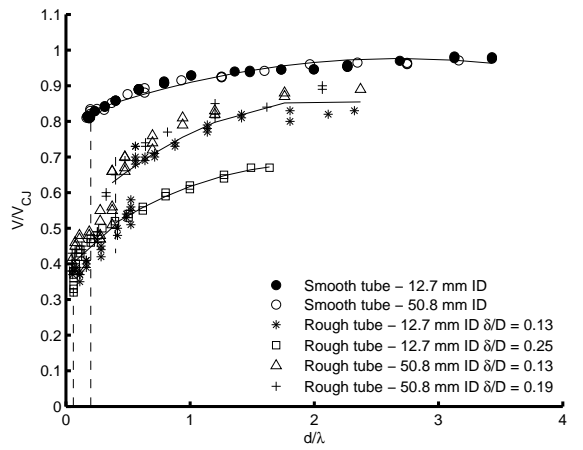

Figure 4: Velocity variation for $\mathrm{C}_{2} \mathrm{H}_{2}$ $+2.5 \mathrm{O}_{2}+70 \% \mathrm{Ar}$ 


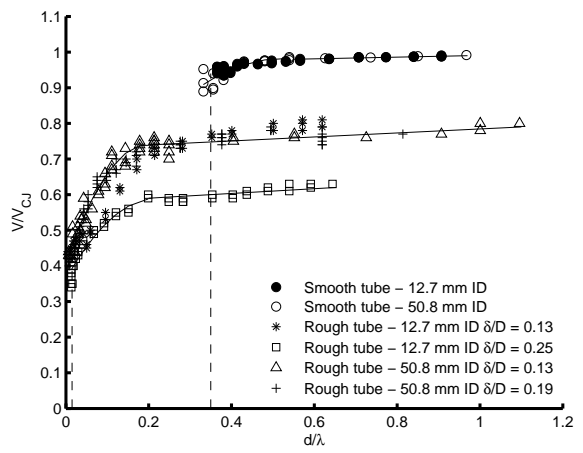

Figure 5: Velocity variation for $\mathrm{CH}_{4}+$ $2 \mathrm{O}_{2}$ 


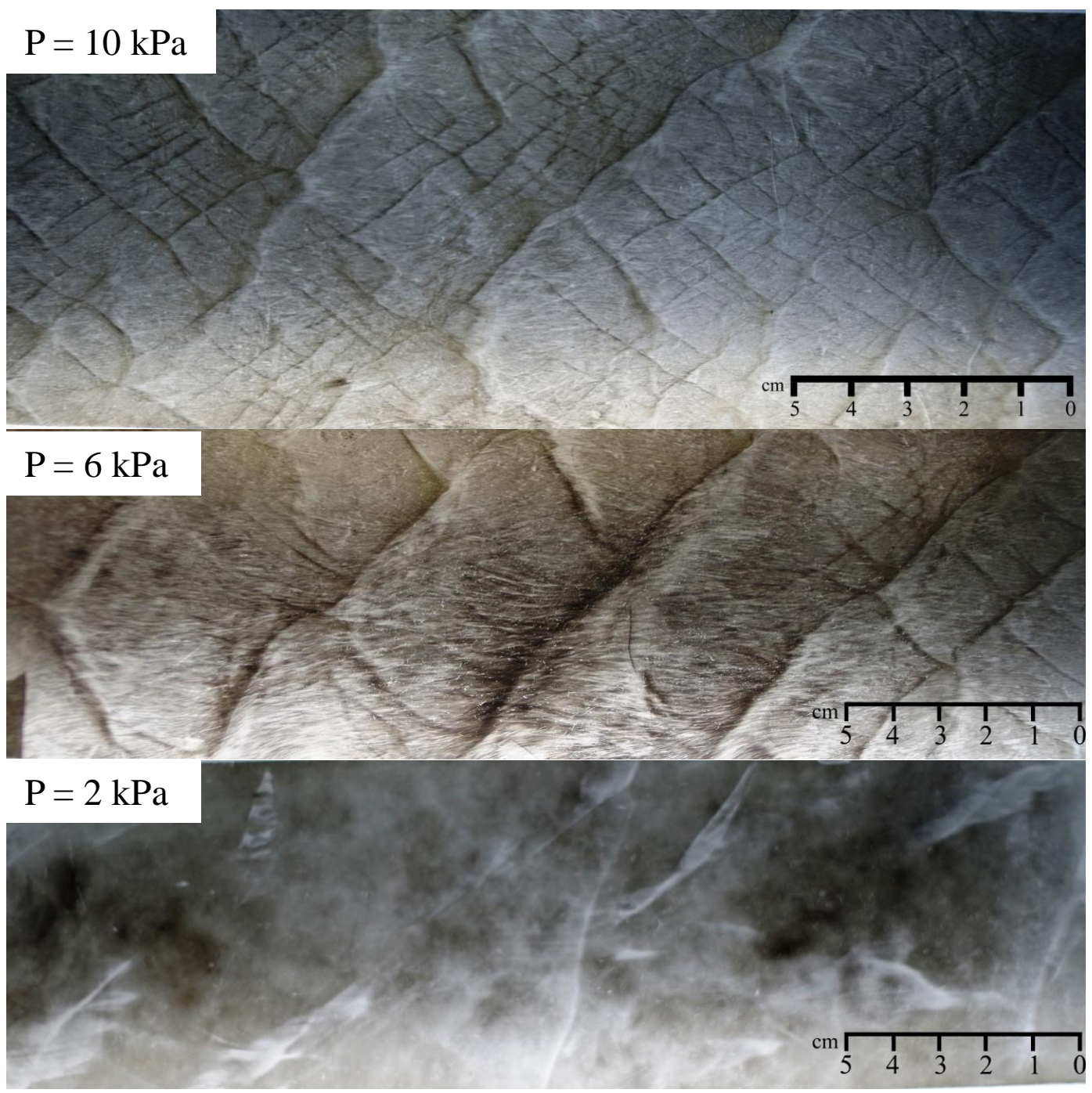

Figure 6: Smoked foils for $\mathrm{C}_{2} \mathrm{H}_{2}+2.5 \mathrm{O}_{2}+70 \% \mathrm{Ar}$ in the $50.8 \mathrm{~mm}$ diameter tube with $\delta / D=0.13$ 


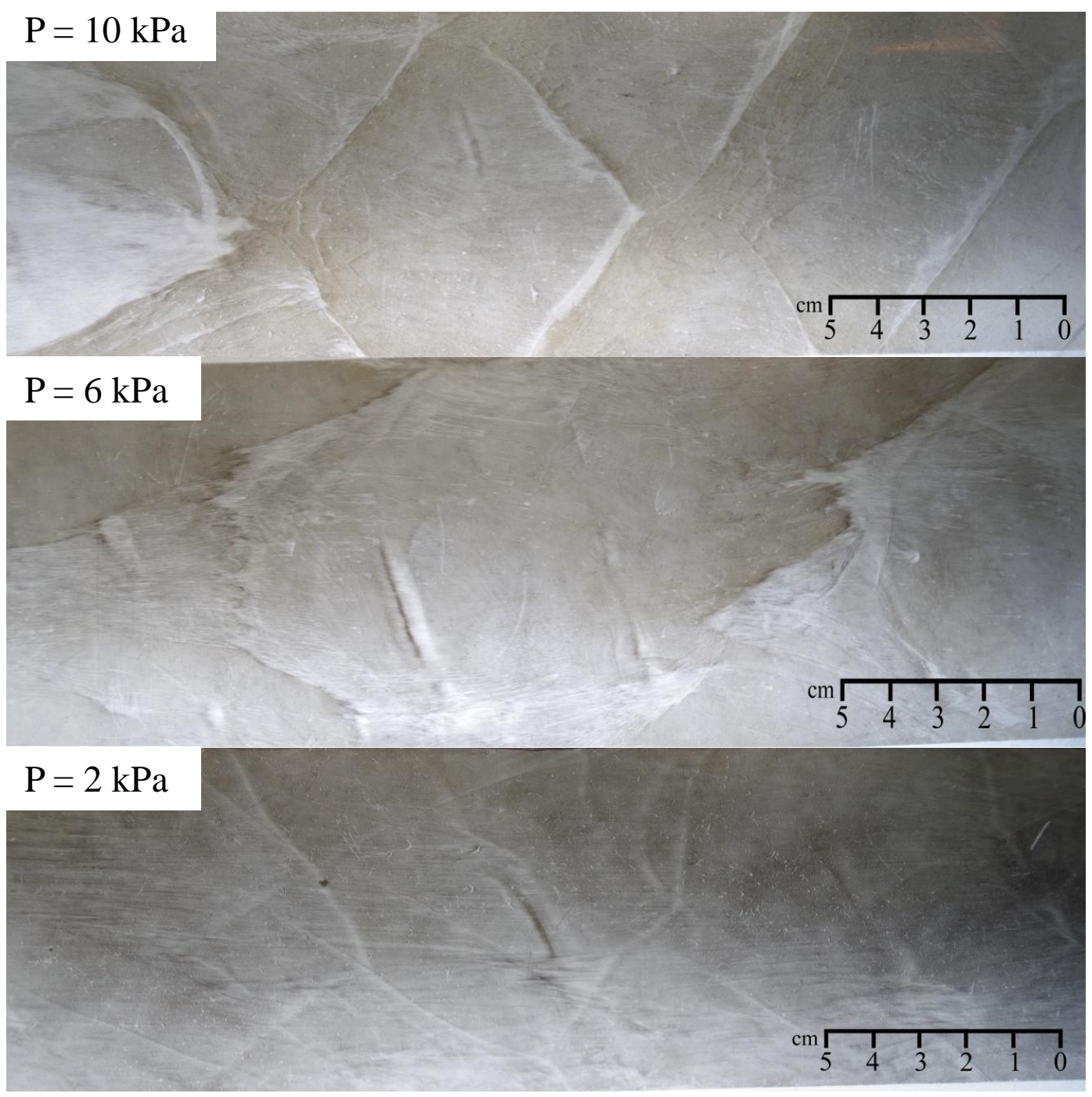

Figure 7: Smoked foils for $\mathrm{CH}_{4}+2 \mathrm{O}_{2}$ in the $50.8 \mathrm{~mm}$ diameter tube with $\delta / D=$ 0.13 


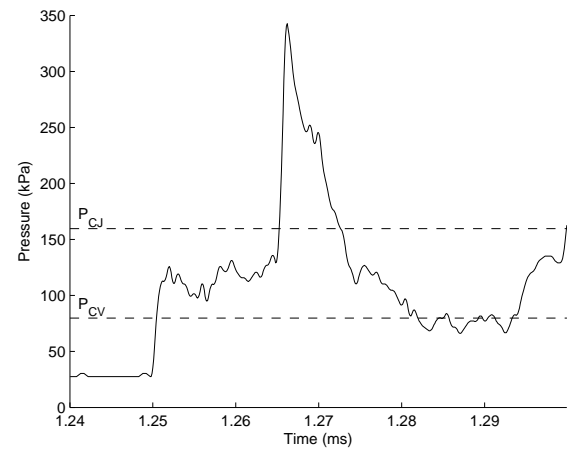

Figure 8: Pressure trace for $\mathrm{CH}_{4}+$ $2 \mathrm{O}_{2}$ in the $50.8 \mathrm{~mm}$ diameter tube with $\delta / D=0.13$ at an initial pressure of $6 \mathrm{kPa}$ 


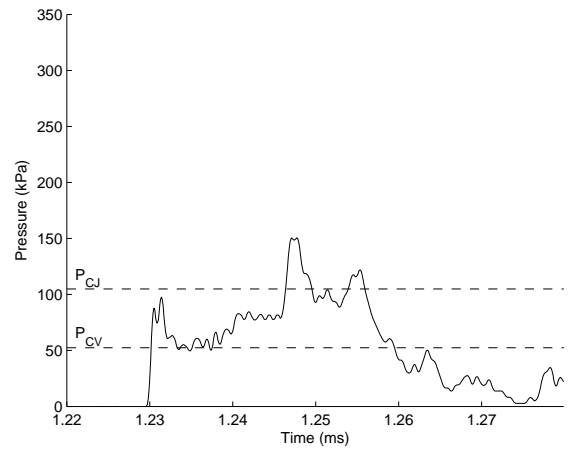

Figure 9: Pressure trace for $\mathrm{CH}_{4}+$ $2 \mathrm{O}_{2}$ in the $50.8 \mathrm{~mm}$ diameter tube with $\delta / D=0.13$ at an initial pressure of $4 \mathrm{kPa}$ 


\section{List of Figures}

Figure 1: Schematic of the experimental setup

Figure 2: Wall roughness

Figure 3: Detonation trajectories for $\mathrm{C}_{2} \mathrm{H}_{2}+2.5 \mathrm{O}_{2}+70 \%$ Ar in the $12.7 \mathrm{~mm}$ diameter tube with

$$
\delta / D=0.13
$$

Figure 4: Velocity variation for $\mathrm{C}_{2} \mathrm{H}_{2}+2.5 \mathrm{O}_{2}+70 \% \mathrm{Ar}$

Figure 5: Velocity variation for $\mathrm{CH}_{4}+2 \mathrm{O}_{2}$

Figure 6: Smoked foils for $\mathrm{C}_{2} \mathrm{H}_{2}+2.5 \mathrm{O}_{2}+70 \%$ Ar in the $50.8 \mathrm{~mm}$ diameter tube with $\delta / D=$ 0.13

Figure 7: Smoked foils for $\mathrm{CH}_{4}+2 \mathrm{O}_{2}$ in the $50.8 \mathrm{~mm}$ diameter tube with $\delta / D=0.13$

Figure 8: Pressure trace for $\mathrm{CH}_{4}+2 \mathrm{O}_{2}$ in the $50.8 \mathrm{~mm}$ diameter tube with $\delta / D=0.13$ at an initial pressure of $6 \mathrm{kPa}$

Figure 9: Pressure trace for $\mathrm{CH}_{4}+2 \mathrm{O}_{2}$ in the $50.8 \mathrm{~mm}$ diameter tube with $\delta / D=0.13$ at an initial pressure of $4 \mathrm{kPa}$ 